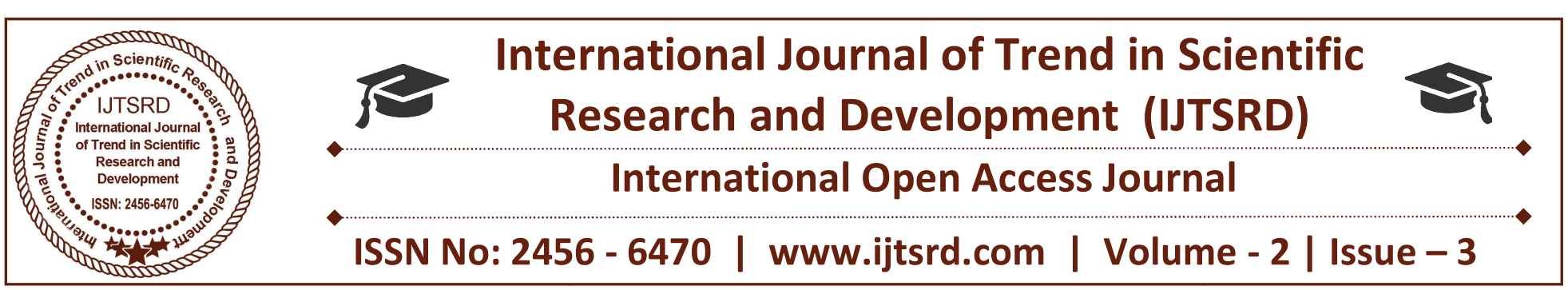

\title{
Automatic Image Captions for Lightly Labelled Images
}

\author{
Raju Janagam, K. Yakub Reddy \\ Assistant Professor, CSE, SVS Group of Institutions, Warangal, Telangana, India
}

\section{ABSTRACT}

We initiate a new distance metric learning technique recognized as ambiguously supervised structural metric learning to find out discriminative Mahalanobis distance metric that is based on weak supervision data. For improving the performance, two affinity matrices are combined to get a fused affinity matrix which is used for face naming. When specified a collection of images, in which each of the image contains numerous faces and is linked by few names in corresponding caption, the purpose of face naming is to infer acceptable name for each face. Here we introduce two methods to correspondingly get hold of two discriminative affinity matrices by means of learning from the images of weakly labelled. For initial affinity matrix obtaining, we put forward a new method known as regularized low-rank representation by incorporation of weakly supervised information into low-rank representation with the intention that affinity matrix is obtained from resulting reconstruction coefficient matrix.

Keywords: Images, Regularized low-rank, Affinity matrices, Face naming, Mahalanobis distance metric

\section{INTRODUCTION:}

In our work we introduce two methods to correspondingly get hold of two discriminative affinity matrices by means of learning from the images of weakly labelled. The two affinity matrices are later combined to produce one combined affinity matrix, on the basis of which an iterative method is developed for the process of automatic face naming [1]. For obtaining of initial affinity matrix, we suggest a new method known as regularized low-rank representation by means of incorporation of weakly supervised information into low-rank representation technique with the intention that affinity matrix is obtained from resulting reconstruction coefficient matrix. In the recent times, there is an increased study carried out in developing of automatic methods for naming of face in images in addition to videos. In our work we make a focus on annotating faces within images that are based on ambiguous supervision from connected captions. Low-rank representation is an unsupervised method for exploring of several subspace data structures. To infer correspondences between faces that are based on visual features and names within candidate name sets, we make use of subspace structures between faces that are based on several assumptions such as the faces from same subject lie within same subspace and subspaces are independent. Our proposed regularized low-rank representation is related to low-rank representation and low-rank support vector machine method. Our regularized low-rank representation is connected to reconstruction basis method low-rank representation.

\section{METHODOLOGY:}

We introduce a novel distance metric learning technique known as ambiguously supervised structural metric learning to find out discriminative Mahalanobis distance metric that is based on weak supervision data. Our ambiguously supervised structural metric learning is on basis of ambiguous supervision. We utilize max margin loss to hold ambiguity of structural output, by means of enforcing distance on the basis of best label assignment matrix in possible label set to be outsized than distance on the basis of top label assignment matrix in infeasible label set by means of a margin [2][3]. On the basis of caption-based weak supervision, we suggest a novel technique regularized low-rank representation by means of introduction of a novel regularizer into lowrank representation and we can analyse the initial affinity matrix by means of resultant reconstruction 
coefficient matrix. On the other hand, we make usage of similarity matrix on the basis of Mahalanobis distances among faces as another affinity matrix. In this technique we make a consideration of constraints for label matrix of faces within each image by means of usage of practicable label set, and we later define image to assignment distance that measure up incompatibility among label matrix and faces from each image on the basis of distance metric. These two affinity matrices are later combined to produce one combined affinity matrix, on the basis of which an iterative method is developed for the process of automatic face naming. While regularized low-rank representation and ambiguously supervised structural metric learning survey weak supervision in various ways and they are both useful, two corresponding affinity matrices are likely to hold complementary as well as discriminative information for face naming. Hence for improvisation of the performance, two affinity matrices are combined to get a fused affinity matrix which is used for face naming. Hence, ambiguously supervised structural metric learning finds a Mahalanobis distance metric that encourage image to assignment distance on the basis of a selected possible label matrix, which estimates ground truth one, to be lesser than the image to assignment distances on the basis of infeasible label matrices to some level.

\section{AN OVERVIEW OF PROPOSED SYSTEMS:}

Low-rank support vector machine method is on the basis of dynamic principal component analysis. Lowrank support vector machine method does not rebuild the data by means of using itself as dictionary. On the contrary, our regularized low-rank representation is connected to reconstruction basis method low-rank representation. Our ambiguously supervised structural metric learning is associated to the works of traditional metric learning [4]. Our ambiguously supervised structural metric learning is on the basis of ambiguous supervision, and we make use of a max margin loss to hold ambiguity of structural output, by means of enforcing distance on the basis of best label assignment matrix in possible label set to be outsized than distance on the basis of top label assignment matrix in infeasible label set by means of a margin. In ambiguously supervised structural metric learning we make a consideration of constraints for label matrix of faces within each image by means of usage of practicable label set, and we later define image to assignment distance that measure up incompatibility among label matrix and faces from each image on the basis of distance metric. Regularized low-rank representation and ambiguously supervised structural metric learning are both useful. The two corresponding affinity matrices are likely to hold complementary as well as discriminative information for face naming. While a similar loss that handles structural output is moreover used in metric learning to rank, it models the ranking orders concerning training samples, and there is no doubt concerning supervision information within metric learning to rank. Our ambiguously supervised structural metric learning is moreover associated to two newly projected approaches for face naming difficulty by means of weak supervision. Multiple-instance logistic discriminant metric learning follows multiinstance learning theory, which assumes that each of the images have to hold a face equivalent to each name within the caption. On the other hand, it might not hold for the problem of face naming as captions are not precise. On the contrary, our ambiguously supervised structural metric learning utilizes a highest margin loss to hold structural output devoid of usage of such assumption. While maximum margin set moreover makes usage of utmost margin loss to manage structural output, maximum margin set aims to find out the classifiers and it was considered for the problem of classification. On the contrary to low-rank representation, our regularized low-rank representation makes usage of weak supervision from image caption and moreover considers constraints of image-level when solving the problem of weakly supervised face naming [5]. Moreover, our regularized low-rank representation differs from lowrank support vector machine method in two aspects such as to make use of weak supervision; low-rank support vector machine method considers the data of weak supervision in partial permutation matrices, whereas regularized low-rank representation make use of our projected regularizer to penalize equivalent reconstruction coefficients. Our ambiguously supervised structural metric learning finds out a metric of distance metric that generates an affinity matrix and is combined by means of affinity matrix from our regularized low-rank representation technique to later get better performance of face naming [6].

\section{CONCLUSION:}

We spotlight on annotating faces within images that are based on ambiguous supervision from connected captions and introduce two methods to correspondingly get hold of two discriminative 
affinity matrices by means of learning from the images of weakly labelled. Our regularized low-rank representation is related to low-rank representation and low-rank support vector machine method. In social networking sites, photo sharing sites as well as news websites, an image that includes several faces are associated by means of a caption that specifying who is in picture. Low-rank representation is an unsupervised method for exploring of several subspace data structures. As regularized low-rank representation and ambiguously supervised structural metric learning survey weak supervision in various ways and they are both useful, two corresponding affinity matrices are likely to hold complementary as well as discriminative information for face naming.

\section{REFERENCES}

1. X. Zhang, L. Zhang, X.-J. Wang, and H.-Y. Shum, "Finding celebrities in billions of web images," IEEE Trans. Multimedia, vol. 14, no. 4, pp. 9951007, Aug. 2012.

2. Z. Zeng et al., "Learning by associating ambiguously labeled images," in Proc. 26th IEEE Conf. Comput. Vis. Pattern Recognit., Portland, OR, USA, Jun. 2013, pp. 708-715.

3. M. Everingham, J. Sivic, and A. Zisserman, "Hello! My name is Buffy-Automatic naming of characters in TV video," in Proc. $17^{\text {th }}$ Brit. Mach. Vis. Conf., Edinburgh, U.K., Sep. 2006, pp. 899908.

4. M.-L. Zhang and Z.-H. Zhou, "M3MIML: A maximum margin method for multi-instance multi-label learning," in Proc. 8th IEEE Int. Conf. Data Mining, Pisa, Italy, Dec. 2008, pp. 688-697.

5. T. Cour, B. Sapp, C. Jordan, and B. Taskar, "Learning from ambiguously labeled images," in Proc. 22nd IEEE Conf. Comput. Vis. Pattern Recognit., Miami, FL, USA, Jun. 2009, pp. 919 926. 